\title{
Anàlisis de ganancia en el caso cuasi-degenerado en un material fotorrefractivo
}

\section{Braiyans Miguel Barrera, Néstor Alonso Arias Hernández, Martha Lucía Molina Prado}

Grupo de óptica moderna, Departamento de Física y Geología, Universidad de Pamplona.

Resumen: Se resuelven las ecuaciones de onda que describen el acoplamiento cuasi-generado que presentan dos haces en un material fotorrefractivo para registros de alta frecuencia. Las ecuaciones de acoplamiento cuasi-generado de dos haces en un material fotorrefractivo dependen del coeficiente de acoplamiento, el tiempo de respuesta del medio, la frecuencia de los dos haces de entrada, el coeficiente de absorción y la relación de las intensidades a la entrada. A su vez, el tiempo de respuesta del medio está en función de la relación de concentración de impurezas, es decir, la relación entre la densidad del número de impurezas aceptoras $\left(\mathrm{N}_{\mathrm{A}}\right)$ y la densidad del número de impurezas donadoras $\left(\mathrm{N}_{\mathrm{D}}\right)$; del campo de difusión, campo de drift o arrastre y campo de saturación. Un análisis teórico detallado del efecto de estos parámetros (razón de concentración, diferencia de frecuencia de oscilación, constante de acoplamiento, etc) en la ganancia se muestra en este trabajo.

Palabras clave: acoplamiento, cuasi-generado, dos haces, material fotorrefractivo

\section{Analysis of gain in quasi-degenerated in photorefractive crystal}

\begin{abstract}
The wave equations that describe the quasi generated coupling between two beams in a photorefractive material for high frequency recording are solved. The quasidegenerated two-beam coupling equations in a photorefractive crystal depend on the coupling coefficient, the response time of the medium, the shift frequency oscillation at the input beams, the absorption coefficient and the input intensity ratio. This response time is function of characteristic time constant of the medium, that is, of the ratio concentration between the density of acceptor impurities $\left(\mathrm{N}_{\mathrm{A}}\right)$ and the density of donor impurities $\left(\mathrm{N}_{\mathrm{D}}\right)$, equally of the diffusion field, the saturation field and drift field. A detailed theoretical analysis of the influence of these parameters (concentration ratio, shift frequency oscillation, coupling constant, etc.) on the gain for the non-degenerate two beam is realized.
\end{abstract}

Key words: quasi generated ,coupling, two beams, photorefractive material 


\section{Introducción}

139

Los materiales fotorrefractivos son materiales foto-conductores electro-ópticos capaces de cambiar sus propiedades bajo incidencia de luz no homogénea. Las propiedades de estos materiales hacen que exista un amplio campo de investigación para desarrollos futuros. El acoplamiento de dos haces en cristales fotorrefractivos ha sido ampliamente estudiado debido a sus posibles aplicaciones en el procesamiento de señales, comunicaciones ópticas, computación óptica, holografía en tiempo real, mejora de imágenes y memorias holográficas[1-10]. La descripción teórica más común del acoplamiento de dos ondas en materiales fotorrefractivos se conoce como la teoría de ondas acopladas [11].

La no uniformidad del patron de luz sobre el cristal, induce dentro del cristal una red de indices de refracción por efecto electro-optico. La red de índices se puede considerar como una red de volumen dinámico, produciendose un acoplamiento no lineal, el cual resulta en transferencia de energía y fase entre los dos haces que están interactuando. La transferencia de energía (acoplamiento) entre los dos haces en el cristal fotorrefractivo tiene lugar debido a un desajuste de fase entre la red de intensidad de luz incidente en el cristal y la red de índices de refracción producida dentro del cristal. La transferencia de energía máxima se da cuando el patron de franjas y la red fotoinducida son ajustadas por $\frac{\pi}{2}$ (en el caso de difusión solamente)[12-14].

La ganancia en el mezclado de dos ondas es un parámetro importante y útil en los materiales fotorrefractivos. En el caso cuasi-degenerado, la ganancia depende del tiempo de respuesta de la red móvil.

En el caso de acoplamiento de dos haces no degenerado, la ganancia no solo depende del espesor del cristal, coeficiente de acoplamiento, coeficiente de absorción y relación de intensidad de entrada, sino también en el cambio de frecuencia de oscilación y el tiempo de respuesta del medio fotorrefractivo. Este tiempo de respuesta es función de la constante de tiempo característica del medio, la cual depende de la relación de concentración, es decir del numero de aceptores $\left(\mathrm{N}_{\mathrm{A}}\right)$ y donores $\left(N_{D}\right)$ del material. En el presente trabajo teóricamente analizamos la ganancia en el caso cuasi-degenerado dentro de los materiales fotorrefractivos que tienen propiedades absorbentes bajo la aproximación de amplitud lentamente variable [11], con respecto a la razón de concentración del material $\left(\mathrm{N}_{\mathrm{D}} / \mathrm{N}_{\mathrm{A}}\right)$ y los parámetros mencionados.

\section{Discusión teórica}

Cuando dos haces de diferente frecuencia inciden en un medio fotorrefractivo el patrón de franjas ya no es estacionario, con diferente frecuencia de los haces, todavía se puede inducir una red de índices de volumen [1] de tal manera, que el patrón de franjas no se mueva demasiado rápido.

Si $w_{1} y w_{2}$ son las frecuencias de los dos haces incidentes, $w_{1}$ del haz de mayor intensidad (haz de bombeo) y $w_{2}$ del haz de menor intensidad (haz señal), podemos escribir el campo eléctrico de los dos haces como: 
139-1

$E_{j}=A_{j} e^{i\left(w_{j} t-\overrightarrow{k_{j}} \cdot \vec{r}\right)} \quad j=1,2$

Donde $A_{1}$ y $A_{2}$ son las amplitudes de los haces y $\vec{k}_{1}$ y $\vec{k}_{2}$ sus vectores de onda. La intensidad de la radiación electromagnética resultante puede escribirse como:

$$
I=A_{1} A_{1}^{*}+A_{2} A_{2}^{*}+A_{1} A_{2}^{*} e^{-i((\Omega t-\vec{K} \cdot \vec{r})}+A_{2} A_{1}^{*} e^{i(\Omega t-\vec{K} \cdot \vec{r})}
$$

Donde $\Omega=w_{2}-w_{1}$ y $\vec{K}=\vec{k}_{2}-\vec{k}_{1}$

La distribución de intensidad dada por Ec. (2) representa un patrón de franjas de interferencia que se mueve con una velocidad:

$$
v=\frac{\Omega}{|\vec{K}|}=\frac{\Omega \Lambda}{2 \pi} \quad K=\frac{2 \pi}{\Lambda}
$$

$\Lambda$ período del patrón de franjas. El índice de refracción incluyendo la red de índices inducida por la distribución de intensidades de la Ec. (2) puede ser escrito como [8]:

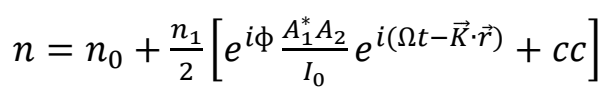

$n_{0}$ índice inicial en ausencia de luz, $\phi$ el grado por el cual la red de índices es cambiada espacialmente con respecto al patrón de interferencia [9]

$$
\begin{aligned}
& \phi=\phi_{0}+\tan ^{-1}(\Omega \tau) \\
& n_{1}=\frac{2 \Delta_{s}}{\sqrt{1+(\Omega \tau)^{2}}}
\end{aligned}
$$

Donde, $\Delta_{S}$ es el valor de saturación del cambio de índice inducido

Los parámetros, $\Delta_{s}$ y $\phi_{0}$ son un cambio de fase constante relacionados con la respuesta no local del cristal bajo la iluminación del patrón de interferencia y dependen no solo del espaciado de la red y su dirección, también de las propiedades del material, por ejemplo, los coeficientes electroópticos, y por ende, del campo eléctrico aplicado. En un medio fotorrefractivo que opera solo por difusión (es decir, sin campo eléctrico externo aplicado), la magnitud de $\phi_{0}$ es igual a $\frac{\pi}{2}[1,11]$.

Sobre la base del modelo de transporte de banda en el que las ecuaciones de velocidad de los materiales se resuelven para el movimiento de la red de índices, bajo la suposición $I_{1} \ll I_{0}$ el tiempo de respuesta $\tau$ viene dado por la relación $[17,18]$ :

$$
\tau=t_{0}\left[\frac{E_{d}+E_{\mu}}{E_{d}+E_{q}}\right] ; \quad t_{0}=\frac{1}{r s I_{0}} ; \quad r=\frac{N_{D}}{N_{A}},
$$

$t_{0}$ es un tiempo constante característico del medio, $s$ sección eficaz, $E_{d}$ Campo de difusión, $K_{B}$ constante de Boltzman, $T$ temperatura, $E_{\mu}$ Campo característico (denominado campo drift), $\gamma_{r}$ 
Constante de recombinación, $N_{A}$ numero de aceptores, $E_{q}$ Campo de saturación, $r=N_{D} / N_{A}$ razón de concentración de impurezas.

Las ecuaciones acopladas para el caso de acoplamiento cuasi-degenerado están dadas por:

$$
\begin{gathered}
\frac{d I_{1}}{d z}=-\frac{\gamma_{0}}{\left(1+\Omega^{2} \tau^{2}\right)}\left[\frac{I_{1} I_{2}}{I_{1}+I_{2}}\right]-I_{1} \sigma \\
\frac{d I_{2}}{d z}=\frac{\gamma_{0}}{\left(1+\Omega^{2} \tau^{2}\right)}\left[\frac{I_{1} I_{2}}{I_{1}+I_{2}}\right]-I_{2} \sigma
\end{gathered}
$$

Donde $\gamma_{0}$ es la constante de acoplamiento para el caso degenerado y $\sigma$ coeficiente de absorción. Al integrar las ecuaciones se obtienen:

$$
\begin{aligned}
& I_{1}(z)=I_{1}(0)\left[\frac{1+M^{-1}}{1+M^{-1} e^{\left(\frac{\gamma_{0}}{\left(1+\Omega^{2} \tau^{2}\right)}\right) z}}\right] e^{-\sigma z} \\
& I_{2}(z)=I_{2}(0)\left[\frac{1+M}{1+M e^{-\frac{-\gamma_{0}}{\left(1+\Omega^{2} \tau^{2}\right)^{z}}}}\right] e^{-\sigma z}
\end{aligned}
$$

$M=\frac{I_{1}(0)}{I_{2}(0)}$ razón de las intensidades a la entrada del cristal.

Se hace evidente que la energía fluye del haz $I_{1}$ hacia el haz $I_{2}$, debido a esto el haz que tiene ganancia energética es $I_{2}$, la ecuación de ganancia para el haz $I_{2}$ tiene la forma:

$$
g_{2}=\frac{1+M}{1+\mathrm{Mexp}\left[\frac{-\gamma_{0} L}{1+\Omega^{2}\left[\frac{1}{r s I_{0}}\left[\frac{E_{d}+E \mu}{E_{d}+E_{q}}\right]\right]^{2}}\right]} \exp (-\sigma L)
$$

\section{Resultados}

Utilizando la ecuación (12) se analiza como varia la ganancia energética a lo largo del cristal. Los resultados se presentan para frecuencias de red de 2000 lineas $/ \mathrm{mm}, \lambda=532 \mathrm{~nm}$ en un cristal $B S O$. Los parámetros físicos utilizados para la simulación de la mezcla cuasi-degenerada de dos ondas son $[1,15,16]$

\begin{tabular}{|c|l|c|}
\hline \multicolumn{2}{|c|}{ Parámetros constantes } \\
\hline$\gamma_{r}$ & Constante de recombinación $\left(\mathrm{cm}^{-3} \mathrm{~s}^{-1}\right)$ & $1.65 \times 10^{-11}$ \\
\hline$\mu_{e}$ & constante de movilidad efectiva $\left(\mathrm{cm}^{2} \mathrm{~V}^{-1}\right)$ & $1 \times 10^{-3}$ \\
\hline$\varepsilon$ & Constante dieléctrica $(\mathrm{BSO})$ & 56 \\
\hline$\varepsilon$ & Constante dieléctrica $\left(\mathrm{BaTiO}_{3}\right)$ & 1000 \\
\hline$N_{A}$ & densidad de aceptores $\left(\mathrm{cm}^{-3}\right)$ & $1.2 \times 10^{16}$ \\
\hline$r_{41}$ & coeficiente electro-óptico efectivo $\left(\mathrm{cm} \mathrm{V}^{-1}\right)$ & $4.7 \times 10^{-10}$ \\
\hline
\end{tabular}




\begin{tabular}{|c|l|c|}
\hline$n_{0}$ & Índice de refracción inicial & 2.5 \\
\hline$\sigma$ & Coeficiente de absorción $\left(\mathrm{cm}^{-1}\right)$ & 1 \\
\hline$S$ & Sección eficaz $\left(\mathrm{cm}^{2} \mathrm{~V}^{-1}\right)$ & $1.06 \times 10^{-1}$ \\
\hline \multicolumn{2}{|c|}{ Parámetros variables } & Desde --- Hasta \\
\hline$\Omega$ & Diferencia de frecuencia $(\mathrm{Hz})$ & $0.1--1$ \\
\hline$N_{D}$ & Densidad de donores $\left(\mathrm{cm}^{-3}\right)$ & $0.5 \times 10^{19}--2 \times 10^{19}$ \\
\hline$\gamma_{0}$ & Constante de acoplamiento $\left(\mathrm{cm}^{-1}\right)$ & $5--25$ \\
\hline
\end{tabular}

Se puede apreciar que a medida que aumenta el espesor del cristal la ganancia es mayor, llegando a un máximo, para la figura 1-(a) ese máximo se presenta para $L \approx 0.45 \mathrm{~cm}$, para la figura 1-(b) en $L \approx 0.68 \mathrm{~cm}$ y para la figura 1 -(c) en $L \approx 0.91 \mathrm{~cm}$. Luego de ese máximo de energía que se presenta, la energía disminuye exponencialmente debido a la absorción que presenta el material a lo largo del cristal. Es apreciable que para valores mayores a $r \approx 500$ la ganancia es independiente de la razón de concentración de impurezas. A medida que se aumenta la modulación $M$, se aumenta la ganancia y el máximo de energía, esto debido a la diferencia que presenta el haz 1 de referencia o de bombeo, con respecto al el haz 2 o haz señal.

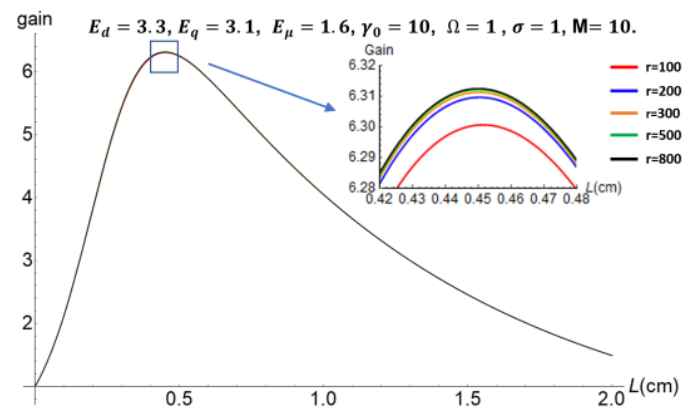

(a)

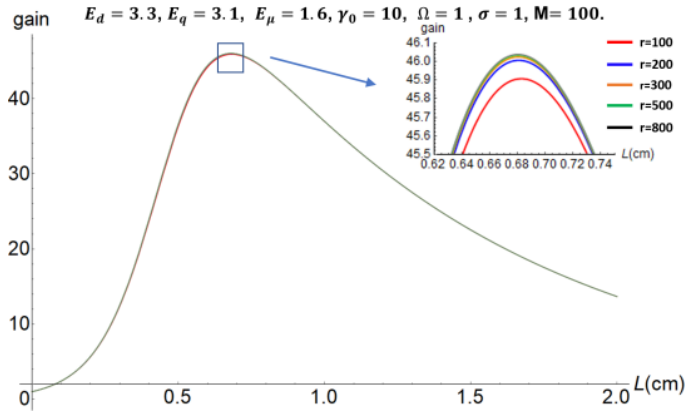

(b)

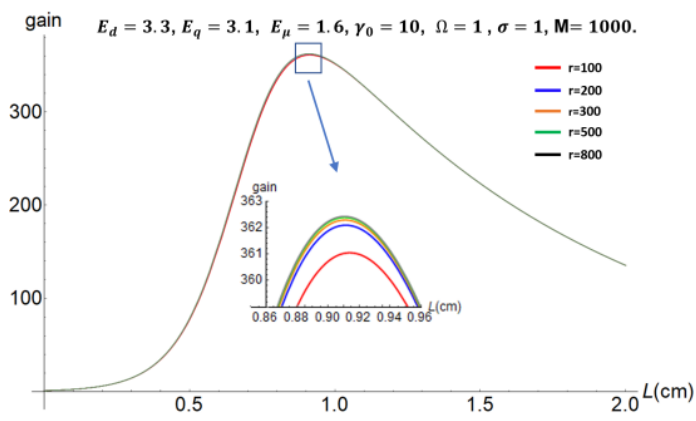

(c)

Figura 1. Variación de la ganancia en la mezcla de dos ondas cuasi-degenerado en cristales fotorrefractivos, en función del espesor del cristal. Para diferentes valores de $r$. Con valores $E_{d}=3.3 \mathrm{kV} / \mathrm{cm}, E_{q}=3.1 \mathrm{kV} / \mathrm{cm}, E_{\mu}=1.6 \mathrm{kV} / \mathrm{cm}$,

$$
\gamma_{0}=10 \mathrm{~cm}^{-1}, \Omega=1 \mathrm{~Hz}, \sigma=1 \mathrm{~cm}^{-1} \text {. (a) } M=10, \quad \text { (b) } M=100, \quad \text { (c) } M=1000
$$


En los resultados presentados en la Figura 2 se puede observar la ganancia energética en función del espesor del cristal, para diferentes valores de $\Omega$. Es evidente que a medida que disminuye la diferencia de oscilación $\Omega$ entre los haces, la ganancia energética se hace mayor, siendo apreciable que para valores menores a $\Omega \approx 0.3 \mathrm{~Hz}$ la ganancia se hace independiente de la diferencia de oscilación entre los haces.

En la Figura 3. Se puede observar la ganancia energética en función del espesor del cristal $L$, para diferentes valores de acoplamiento $\gamma_{0}$. Se puede apreciar que a mayor constante de acoplamiento $\gamma_{0}$ que exista entre los haces, la ganancia es mayor y el máximo de energía (energía optima de transferencia) se presenta para cristales de menor espesor. Siendo $\gamma_{0}$ una constante proporcional a la ganancia.

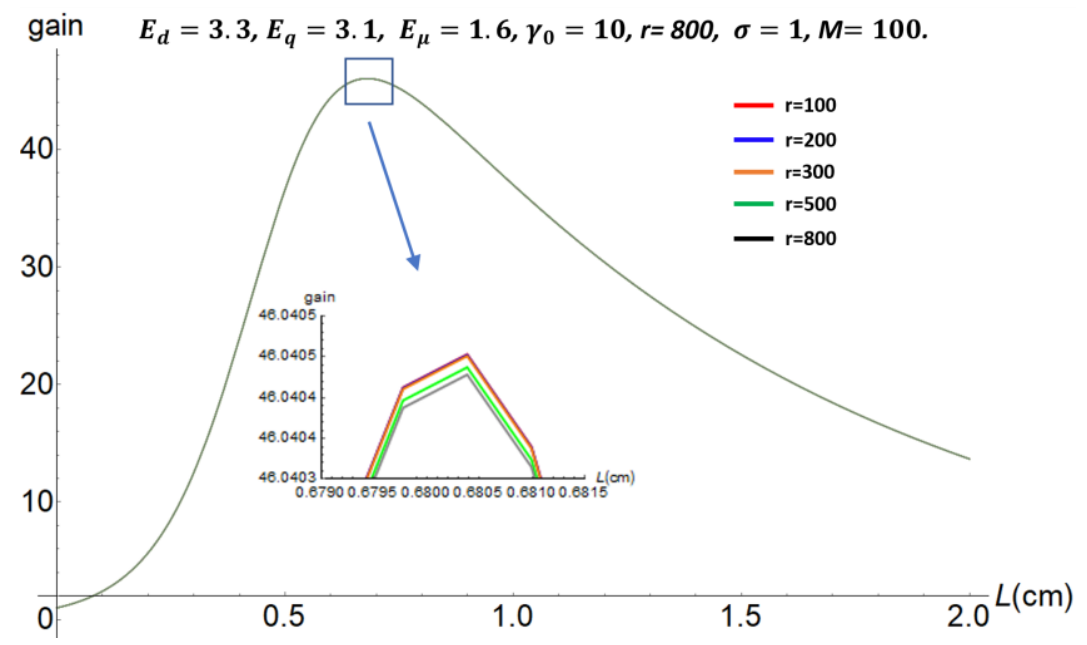

Figura 2. Variación de la ganancia en la mezcla de dos ondas cuasi-degenerado en cristales fotorrefractivos, en función del espesor del cristal. Para diferentes valores de $\Omega$. Con valores $E_{d}=3.3 \mathrm{kV} / \mathrm{cm}, E_{q}=3.1 \mathrm{kV} / \mathrm{cm}, E_{\mu}=1.6 \mathrm{kV} / \mathrm{cm}$, $\gamma_{0}=10 \mathrm{~cm}^{-1}, \sigma=1 \mathrm{~cm}^{-1}, M=100$

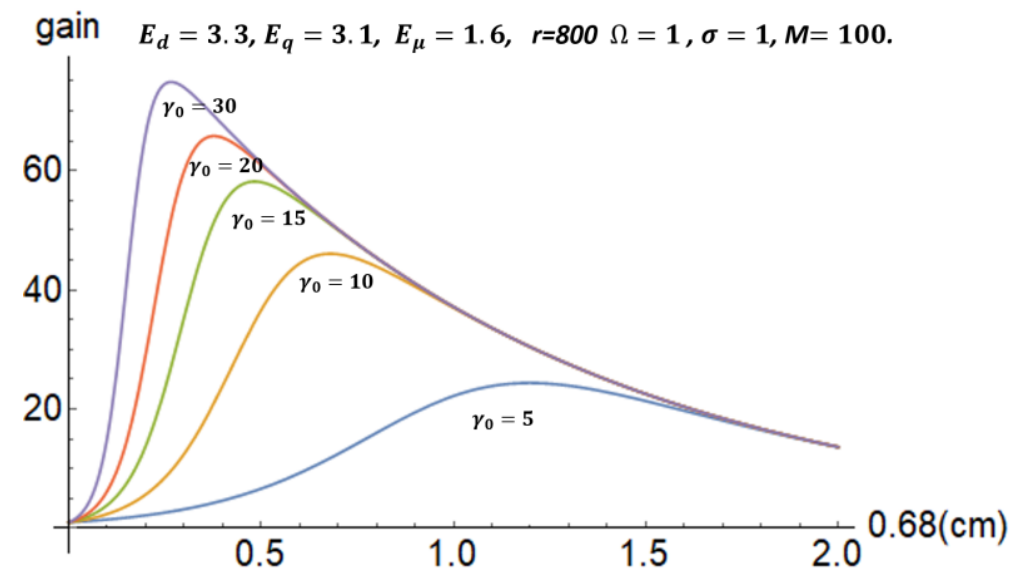


Figura 3. Variación de la ganancia en la mezcla de dos ondas cuasi-degenerado en cristales fotorrefractivos, en función del espesor del cristal. Para diferentes valores de $\gamma_{0}$. Con valores $E_{d}=3.3 \mathrm{kV} / \mathrm{cm}, E_{q}=3.1 \mathrm{kV} / \mathrm{cm}, E_{\mu}=1.6 \mathrm{kV} / \mathrm{cm}$, $\mathrm{r}=800, \sigma=1 \mathrm{~cm}^{-1}, M=100$ y $\Omega=1$

Finalmente, en los resultados presentados en la Figura 4. Se puede observar la ganancia energética en función de la razón de concentración $r$, tanto para un cristal $\mathrm{BSO}$ como para un $\mathrm{BaTiO}_{3}$. En la gráfica se observa que para el cristal $B S O$ la ganancia energética es mayor que la ganancia para un cristal $\mathrm{BaTiO}_{3}$, esto se debe a que la constante dieléctrica efectiva es menor para el cristal $\mathrm{BSO}$, como resultado de ello el campo de saturación del cristal $B S O$ es mayor que el campo de saturación del cristal $\mathrm{BaTiO}_{3}$. También se puede apreciar que la ganancia es independiente de la razón de concentración $r$ para un valor de $r \approx 800$ para los dos cristales.

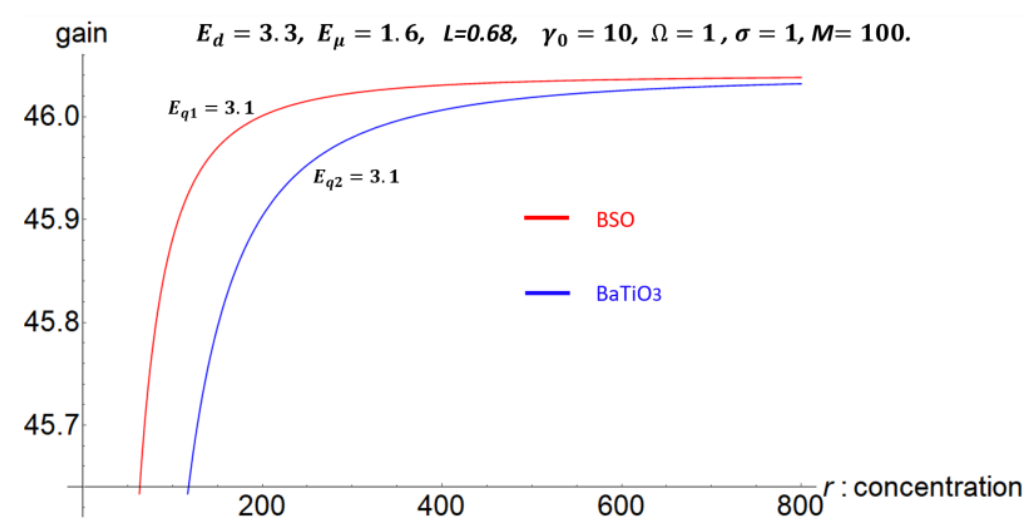

Figura 4. Variación de la ganancia en función de la razón de concentración para un cristal $\mathrm{BSO}$ y un $\mathrm{BaTiO}_{3}$. Con valores $E_{d}=3.3 \mathrm{kV} / \mathrm{cm}, E_{q}=3.1 \mathrm{kV} / \mathrm{cm}, E_{\mu}=1.6 \mathrm{kV} / \mathrm{cm}, \mathrm{L}=0.68, \sigma=1 \mathrm{~cm}^{-1}, M=100 \mathrm{y} \Omega=1$

\section{Conclusiones}

La ganancia en la mezcla cuasi-degenerada de dos ondas en materiales fotorrefractivos es un parámetro importante y útil, y no es función sólo del coeficiente de acoplamiento de energía, el grosor del cristal, de la relación de modulación, coeficiente de absorción, sino también es función de la razón de concentración $r=\frac{N D}{N A}$ y el cambio de frecuencia de oscilación $\Omega$. La ganancia aumenta con el aumento de la relación de concentración $r=\frac{N D}{N A}$ y se observa que la ganancia es mayor para el cristal $\mathrm{BSO}$ a valores más bajos de relación de concentración que la del cristal $\mathrm{BaTiO}_{3}$. Por lo tanto, la respuesta del cristal $\mathrm{BSO}$ es mejor que la respuesta del cristal $\mathrm{BaTiO}_{3}$, en el caso cuasi-degenerado.

\section{Agradecimientos}

Esta investigación fue realizada bajo el proyecto: PR 130-00-001 (GA160-CM-I-2014-2.1.2.2.1)Unipamplona. Martha Lucía Molina Prado, agradece el soporte financiero dado por la Universidad de Pamplona, a traves de su Vicerrectoría de Investigaciones para la realización de dicho trabajo. 


\section{Referencias Bibliográficas}

[1] Yeh, "Introduction to Photorefractive Nonlinear Optics," Wiley, New York, (1993)

[2] L.F. Magaña, I. Casar, J.G. Murillo, "Beam energy exchange in sillenite crystals (Bi12SiO20 and Bi12TiO20), considering the variation of light modulation along sample thickness in a strong nonlinear regime," Opt. Mater. 30, 979-986 (2008).

[3] Marrachi, A., Johnson, R.V, Tanguay, Jr.A.R "Polarization properties of photorefractive diffraction in electrooptic and optically active sillenite crystals (Bragg Regime), J. Opt. Som. A. B, (1986)

[4] Gonzales, G., Zuñiga, A. "Optimization of the diffraction efficiency in nonuniform gratings in sillenite crystals considering the variation of fringe period, optical activity and polarization angles in a strong non-linear regime," Revista Mexicana de Física, (2009)

[5] Molina Prado M.L., et al. "Optimization of the diffraction efficiency in photorefractive crystals" Opt. Pura Apl. 50 (2) 181-186 (2017)

[6] Molina M.L. et al, "Eficiencia de difracción del registro de speckle modulados generados a partir de superficies reflecto-difusoras," Bistua, 10 (2), 2012.

[7] Refregier P, Solymar L, Rajbenbach and Huignard J. P. "Two-beam coupling in photorefractive crystals with moving grating". Appl. Phys. 58. 45-57. (1985).

[8] Fischer B, Cronin-Golomb M, White J O and Yariv A, "Amplified reflection, transmission, and selfoscillation in real-time holography", 1981 Opt Lett 6519

[9] L. Mosquera, I. de Oliveira, J. Frejich, A.C. Hernandes, S. Lanfredi, J.F. Carvalho, "conductivity, photoconductivity, and light-induced absorption in photorefractive sillenite crystals"; J. Appl. Phys. 90 (2001) 2635-2641.

[10] H. Rajbenbach, I.P. Huignard and B. Loiseaux. "Spatial frecuency dependence of the energy transfer in two-wave mixing experiments with BSO crystals", Opt. commun 48(1983) 247-252.

[11] N.V. Kukhtarev, V.B. Markov, S.G. Odulov, M.S. Soskin, V.L. Vinetskii," "Holographic storage in electro-optic crystals" Ferroelecrics. 22 (1979) 961.

[12] D.L.J. Staebler, J. Amodei, “Coupled-wave Analysis of holographic storage in LiNbO3”, Appl. Phys 43 (1972) 1042-1049.

[13] N. Khelfaoui, D. Wolfersberger, G. Kugel, N. Fressengeas, M. Chauvet, "Temporal behavior of two-wave-mixing in photorefractive InP:Fe versus temperature"; Opt. Commun. 261 (2006) 169174.

[14] J.S. Liu et al, "Holographic solitons in photorefractive dissipative systems"; Opt. Lett. 28 (2003) pag 2237. 
[15] Yadav T.K, M, K Maurya, R. A Yadav. "Dynamic study of the gain in two beam coupling in photorefractive materials". Optik 123(2012) pag 1329-1335.

[16] P.Gunter, J.-P. Huignard. "Photorefractive materials and their applications I fundamental phenomena". Springer-Verlag. (1988).

[17] C.H. Kwak, S.Y. Park, J.S. Jeong, H.H. Suh, E.H. Lee, “An analytical solution for large modulation effects in photorefractive two-wave couplings", Opt. Commun. 105 (1994) 353-358.

[18] C.H. Kwak, S.Y. Park, E.H. Lee, "Intensity dependent two-wave mixing at large modulation depth in photorefractive BaTi03 crystal"; Opt. Commun. 115 (1995) 315-322.

*Para citar este artículo:Barrera B.M.; Arias Hernández N.A.;Molina Prado M.L. Analysis of gain in quasi-degenerated in photorefractive cristal.Revista Bistua.2018.16(1):138-144

+ Autor para el envió de correspondencia y la solicitud de las separatas: Barrera B.M Grupo de óptica moderna, Departamento de Física y Geología, Universidad de Pamplona. brayansbarrera-5000@hotmail.com

Recibido: Noviembre 20 de 2016

Aceptado: Febrero 07 de 2018 